Questions vives

\section{Questions Vives}

Recherches en éducation

$N^{\circ} 29$ | 2018

La bienveillance en éducation : approches

compréhensives et critiques

\title{
Construire la relation éducative : postulat d'éducabilité, bienveillance et altruisme
}

\section{Tommy Terraz et Amandine Denimal}

\section{(2) OpenEdition}

\section{Journals}

Édition électronique

URL : http://journals.openedition.org/questionsvives/3409

DOI : 10.4000/questionsvives.3409

ISSN : 1775-433X

Éditeur

Université Aix-Marseille (AMU)

Édition imprimée

ISBN : 978-2-912643-53-7

ISSN : 1635-4079

Référence électronique

Tommy Terraz et Amandine Denimal, « Construire la relation éducative : postulat d'éducabilité,

bienveillance et altruisme », Questions Vives [En ligne], № 29 | 2018, mis en ligne le 19 décembre 2018, consulté le 10 décembre 2020. URL : http://journals.openedition.org/questionsvives/3409 ; DOI : https://doi.org/10.4000/questionsvives.3409

Ce document a été généré automatiquement le 10 décembre 2020.

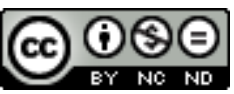

Questions Vives est mis à disposition selon les termes de la licence Creative Commons Attribution -

Pas d'Utilisation Commerciale - Pas de Modification 4.0 International. 


\title{
Construire la relation éducative : postulat d'éducabilité, bienveillance et altruisme
}

\author{
Tommy Terraz et Amandine Denimal
}

\section{Introduction}

1 Tout en s'inscrivant dans la continuité de précédents travaux sur la bienveillance et l'altruisme en éducation ${ }^{1}$, cette étude se propose d'apporter un éclairage singulier à propos de l'éthique de la relation éducative, à partir d'un questionnement sur le postulat d'éducabilité. L'objet de nos analyses philosophiques, réflexives et non exhaustives, est la posture éthique de l'éducateur, sa manière d'être en relation avec, par et pour les sujets éduqués, en vue d'instaurer une relation véritablement éducative, c'est-à-dire qui facilite l'émergence ${ }^{2}$ du sujet éduqué comme personne dans ses dimensions plurielles. Notre réflexion consiste ici à examiner la question de la bienveillance éducative au regard de son articulation avec le postulat d'éducabilité, et ce à partir d'une démarche philosophique qui propose d'aider à repérer les confusions et tensions potentielles existant autour de chacune de ces notions. Pourquoi s'interroger sur l'articulation entre le postulat d'éducabilité et la bienveillance éducative? Parce que ces deux dimensions pourraient être des points d'ancrage, certes non suffisants mais éminemment nécessaires, pour construire une relation éducative.

2 La théorisation contemporaine du postulat d'éducabilité est souvent associée au travail de Philippe Meirieu, qui souligne que l'éducabilité de l'élève est « une postulation fondatrice de la possibilité même d'éduquer, et cela simplement d'abord du point de vue logique. Sans cette postulation, l'entreprise serait totalement dérisoire, complètement vaine et, plus radicalement, impossible » (1991/2016, p. 25). Le postulat d'éducabilité repose sur la croyance, chez l'éducateur, que tout sujet dont il a la responsabilité peut être éduqué, qu'il peut apprendre, progresser - et s'émanciper comme personne relationnelle, aussi libre, autonome et heureuse que possible. Ce postulat, par définition non observable, non 
mesurable et non quantifiable, demande de créditer de façon aprioriste et gratuite le sujet comme éducable ; une démarche au rebours des dynamiques économiques mondiales, qui ont tendance à concevoir les éduqués comme des placements pour l'avenir, des capitaux à faire fructifier. L'éducabilité, si elle est postulée de façon universelle et inconditionnelle, ne présuppose ni n'attend de résultat ni de progrès obligatoire; elle repose au contraire, pour pouvoir s'exercer, sur un renoncement vis-à-vis de l'attente personnelle démesurée de la réussite de l'autre, donc sur un certain décentrement de l'éducateur par rapport à son ego.

3 Cependant, pour que ce postulat soit mis en acte et pour qu'il puisse n'exclure personne, la notion de bienveillance, si intéressante et féconde soit-elle, ne nous semble pas suffisante, et nous expliquerons en quoi le concept d'altruisme semble préférable. Précisons au passage que ces questionnements n'ont pas encore, à notre connaissance, été établis ni travaillés de manière spécifique et explicite en sciences ou en philosophie de l'éducation ${ }^{3}$.

Notre démarche philosophique, logique et analytique, s'applique à interroger ce que l'on entend par relation éducative ${ }^{4}$ quant à ses conditions, ses valeurs et ses finalités. Conformément au mode d'interrogation du texte philosophique, c'est-à-dire l'« interrogation radicale » (Jacques, 2007) ${ }^{5}$, notre posture épistémologique est réflexive, spéculative et non moraliste. Condition n'est pas conditionnement; il s'agit de proposer des repères potentiellement universalisables pouvant avoir une portée praxéologique. Invitant à ne pas délaisser toute préoccupation pour l'universalité en éducation, AnneMarie Drouin-Hans $(1998,2004)$ propose de s'interroger sur les valeurs pouvant être postulées comme universelles, dans une visée de non-nuisance à autrui, quel que soit le contexte: des "universalisables ", "qui ont précisément pour fonction de fonder un humanisme qui soit un dépassement de la condition de pure nature » $(1998$, p. 56). La philosophie de l'éducation ne consiste pas à prescrire ce qu'il faut faire, mais plutôt à se demander pourquoi il semble légitime de pouvoir agir selon telle ou telle direction.

5 Autrement dit, loin de chercher à imposer la bienveillance aux éducateurs ou à leur désigner ce qu'ils doivent désirer (perspective non seulement moralisatrice et paternaliste, mais surtout impossible), nous aurons plutôt à cœur de partager ces repères avec les praticiens qui s'y intéressent, ainsi que le présent mode d'interrogation. Nous espérons dans le même mouvement pouvoir inspirer les recherches scientifiques sur l'éducation. En effet, la démarche scientifique, qui consiste notamment en la description, l'explication et la compréhension du phénomène éducatif, y compris dans ses distorsions, a précisément besoin de pouvoir distinguer ce qui relève de la distorsion, et à ce titre de disposer d'un cadre réflexif de référence établi de façon aprioriste et offrant certains guides d'action à repréciser et à réinterroger sans cesse.

6 Après avoir clarifié les points de rencontre et de distinction entre les notions de bienveillance et d'altruisme, notre tentative de démonstration, en suivant la logique du syllogisme, s'établira en trois temps : (prémisse A) Le postulat d'éducabilité est nécessaire mais non suffisant pour construire une relation éducative; or, (prémisse B) l'altruisme permet au postulat d'éducabilité de se mettre en mouvement de façon juste et bonne, de passer du désir à l'action d'éduquer; donc (conclusion) l'altruisme est nécessaire mais non suffisant pour construire une relation éducative. 


\section{De la bienveillance à l'altruisme : éclairage contextuel et clarifications conceptuelles}

7 Bien que de nombreux courants philosophiques, spirituels et pédagogiques insistent sur l' idée de bienveillance depuis plus de deux millénaires, en Occident comme partout dans le monde, l'engouement récent pour cette notion dans les politiques éducatives françaises, ou encore québécoises, est vraisemblablement en partie lié à l'influence des éthiques du care issues du monde anglo-saxon. Cette fortune nouvelle semble en même temps témoigner d'un retour des préoccupations éthiques au sein des institutions éducatives, qui dans ce mouvement prennent conscience qu'elles ne peuvent se passer des éducateurs en tant qu'agents moraux.

8 Parallèlement, plusieurs études scientifiques portant spécifiquement sur la relation enseignant-élève vont dans ce sens, et montrent que les enseignants qui sont reconnus par leurs élèves comme ayant certaines qualités humaines et vertus éthiques personnelles et professionnelles (comme le respect, la communication empathique, la bienveillance, l'authenticité, la sollicitude, la considération positive) sont les mieux perçus par leurs élèves, qu'ils favorisent une amélioration de leur niveau scolaire, qu'ils permettent de travailler dans une atmosphère générale sereine et pacifiée, et qu'ils favorisent la transmission des valeurs morales et des connaissances en général (voir par exemple Aspy \& Roebuck, 1990; Jennings \& Greenberg, 2009). La bienveillance nous semble donc éminemment intéressante et porteuse dans le champ éducatif; pour autant, est-elle véritablement suffisante pour caractériser la relation éducative dans ses aspects théoriques et pratiques, et plus précisément par rapport au postulat d'éducabilité précédemment évoqué?

9 Avant toute chose, force est de constater qu'il existe des risques de confusion avec d'autres termes à première vue proches. Ainsi, une étude portant sur les discours politiques de la bienveillance éducative et sur les enjeux éthiques et philosophiques associés (Terraz, 2017a) a permis de mettre en évidence que cette notion, qui apparaît depuis les années 2010 dans certains textes officiels de l'Éducation nationale, est souvent envisagée de façon hétérogène. Nous avons notamment émis l'hypothèse que, même si la multiplication des usages de la "bienveillance » atteste sans doute d'une véritable prise de conscience de son importance au-delà du simple phénomène de mode (médiatique, politique, scientifique, etc.), elle ne pourrait prendre forme qu'à travers une éthique, qui trouve ses fondements dans l'expérience et la réflexion personnelles de l'éducateur, considéré en tant qu'agent moral responsable de ses actes. Car on ne peut imposer à quiconque d'être bienveillant, fût-ce par des prescriptions juridiques et institutionnelles : un tel mouvement ne peut venir que de l'agent moral lui-même, qui reconnaît l'intérêt d'être bienveillant, et décide lui-même d'y tendre et de s'y exercer. Dès lors, il nous incombe d'interroger à nouveaux frais la question du désir d'éduquer, et de développer une conception dynamique et relationnelle de la bienveillance.

10 Nous allons à présent tâcher de voir vers quel « autre » la bienveillance est tournée, et si la conception de cet autre est suffisamment inclusive pour ne laisser aucun des sujets éduqués sur le bord du chemin ; parallèlement, nous essayerons de voir si la bienveillance est suffisamment tournée vers l'action concrète. L'exploration présentée ci-dessous prend la forme d'une articulation avec le concept d'altruisme tel que nous le développons dans 
nos travaux (par exemple Terraz, 2016, 2017a/b, 2018a/c), et vient compléter la bienveillance en vue d'élaborer une éthique de la relation éducative.

11 Premièrement, le Centre national de ressources textuelles et lexicales définit notamment la bienveillance comme "disposition favorable d'un supérieur envers un inférieur ${ }^{6}$ ", ce qui n'est pas sans questionner la relation à l'autre qui s'en trouve instaurée ainsi que sa portée. L'altruisme peut en être distingué non seulement en ce qu'il évince cette idée de "supériorité », mais encore en ce qu'il s'adresse à l'autre qui nous est proche, comme à l'autre lointain ou absent. Nous proposons le concept d' " autrui universel » pour insister sur le caractère inconditionnel de la prise en compte de tous les êtres sensibles sans distinction: autant d'hier, que d'aujourd'hui et de demain; aussi bien soi-même qu'autrui ; incluant l'autre singulier, comme alter, et l'autre comme «tiers personnel ${ }^{7}$ »; comme «je ", comme " tu » et comme «il(s)/elle(s)/eux», l'ami comme l'ennemi; le proche comme le lointain; le présent comme l'absent; les Neô̂, c'est-à-dire ceux qui potentiellement peuvent advenir ; bref, tous les êtres humains sans exception et même, autant que possible, les êtres sensibles non humains. Dirigé vers l'autrui universel, l'altruisme s'adresse donc aussi à soi-même car comment pourrais-je prendre soin d'autrui si je me néglige moi-même? Ainsi, l'altruisme se présente comme le plus haut degré d'intensité de bienveillance inconditionnelle et désintéressée. Là où la bienveillance est une ouverture d'esprit empreinte de tolérance et de respect pour l'autre, dans sa liberté et son bien-être, consistant, écrit Kant, à éviter l'indifférence à l'égard d'autrui (1797/1994, p. 317), l'altruisme engage davantage l'agent moral, de façon inconditionnelle et désintéressée donc, tout en étant intimement lié à l'action juste et bonne. À ce titre, il est assez proche des termes agapè et philanthropia des philosophes grecs.

Deuxièmement, là où la bienveillance est d'abord une "disposition favorable envers quelqu'un » (Godin, 2004, p. 149), autrement dit une bien-veillance qui consiste à veiller au bien de l'autre, l'altruisme implique d'emblée la disposition bienveillante envers l' autrui universel, comme nous l'avons dit, mais aussi sa mise en œuvre pratique et effective. En effet, si la bienveillance est appelée à être complétée par la bienfaisance, elle pourrait toutefois aussi se cantonner au seul stade de l'intention sans forcément être mise en œuvre de façon concrète dans l'action. L'altruisme est une vertu morale (envisagée de façon non substantialiste d'un point de vue en ontologie) à la fois humble et discrète ${ }^{8}$ qui se situe au-delà de la simple attitude déclarative ou de façade, et qui consiste à agir. Il s'agit d'une valeur morale choisie de manière consciente, libre et réfléchie par un agent moral qui la reconnaît comme étant bénéfique pour soi-même comme pour autrui, qui y adhère (en tant que cette valeur vaut, est désirable, donne du sens, représente un repère, un guide d'action), inséparable de sa mise en action concrète à travers une intention qui colore l'action juste et bonne ; en tant que telle, elle représente une tentative motivée d'incarnation personnelle de la valeur choisie dans la singularité des situations et des dilemmes éthiques. Là où la bienveillance comporte potentiellement une dimension statique et fixiste, l'altruisme est d'emblée tourné vers autrui et vers l'agir praxéologique; c'est un mouvement relationnel vers l'autre, il est de l'ordre d'une intention dynamique que l'autrui universel puisse être libre intérieurement et heureux : cette intention va colorer les actes de la personne. Ainsi, nous considérons l'altruisme en tant que conception inconditionnelle et actée de la bienveillance : même s'il n'attend rien en retour, l'altruisme est fondamentalement lié au contentement car il amène spontanément à se réjouir de la liberté et du bonheur d'autrui. 
13 Nous pensons donc qu'une éthique de l'altruisme peut contribuer à donner corps à l'attitude bienveillante, à la compléter en en augmentant la portée et la valeur d'acte. Et pour ciseler davantage ce concept, nous allons maintenant préciser ce que l'altruisme n'est pas. D'abord, insistons sur le fait que l'altruisme ne saurait en aucun cas être confondu avec l'amour éros. L'altruisme ne relève ni de la fusion affective, ni de l'attachement, ni de la séduction, ni de l'attente d'une quelconque réciproque à l'égard d'autrui. Il ne cherche pas à capter l'autre ou à le posséder, il n'est pas centré sur soimême mais sur autrui. Inconditionnel et désintéressé, il amène l'éducateur à trouver une juste et bonne distance dans la relation éducative, entre proximité et mise en retrait, jusqu'à ce que le sujet éduqué puisse être le plus autonome et libre possible. Il amène aussi à considérer l'asymétrie de la relation éducative (asymétrie ne concernant que les savoirs et les compétences des acteurs, et non les Personnes dans leur dignité) comme étant temporaire, provisoire, et potentiellement réversible. L'altruisme est donc toujours associé à une intelligence des situations, à une sagesse du discernement.

De plus, il est fondamental de souligner que l'altruisme ne saurait être confondu avec le laxisme, le laisser-faire, l'indifférence ou encore la complaisance ; il relève plutôt d'une juste exigence, et peut tout à fait s'accorder avec une certaine fermeté bienveillante lorsque nécessaire, toujours dans le respect inconditionnel de la Personne, la non-nuisance et la non-violence. Dans la relation éducative, l'altruisme consiste aussi à savoir poser des limites, des normes et des interdits, et à les faire respecter de façon non violente, en vue d'offrir un cadre structurant pour favoriser l'émergence relationnelle de la personne. Car laisser le sujet éduqué en totale " autonomie », lui laisser choisir librement l'intégralité de ses apprentissages, serait faire preuve d'une certaine violence, fût-elle non souhaitée. Éduquer, comme enseigner (insignare en latin), c'est faire signe, indiquer ce qui est souhaitable, préférable ; c'est aussi en donnant des repères, des limites, des directions à suivre, en permettant la co-construction et la transmission d'un héritage culturel et symbolique tout à la fois singulier et universel - celui de l'humanité ${ }^{9}$-, que l'éducateur fait preuve d'altruisme, car c'est ainsi qu'il favorise la structuration du sujet et l'émergence de la personne. Loin d'être en contradiction avec l'autorité éducative - au sens de l'étymologie auctoritas, autorité qui est librement reconnue comme légitime par le sujet éduqué et qui autorise son émancipation - l'altruisme en est au contraire une condition fondamentale (Terraz, 2017b). En outre, l'altruisme semble être une condition de l'instauration d'une relation interlocutive de "dialogue» qui permet la coconstruction non seulement des personnes, mais aussi des significations et des savoirs (Terraz, 2017c, 2018c, à partir de Jacques, 1982). Les savoirs co-construits peuvent alors être articulés entre eux avec finesse sans tomber dans le piège de la confusion indifférenciatrice (Jacques, 2007).

15 Précisons enfin que l'altruisme n'est pas non plus de l'ordre de l'abnégation ni du sacrifice, il est en lien avec la joie, le bonheur, le contentement. Il ne relève pas non plus d'une essence ou d'une substance, il n'est pas inaccessible ou réservé à certaines personnes. En accord avec de nombreuses études scientifiques en psychologie et en neurosciences ${ }^{10}$, il semblerait que chaque être humain dispose d'un potentiel d'altruisme qu'il est possible d'actualiser en s'y exerçant de façon régulière. 


\section{[Prémisse $A]$ Le postulat d'éducabilité est nécessaire mais non suffisant pour construire une relation éducative}

Après avoir réalisé ce travail de clarification concernant les notions de bienveillance et d'altruisme, il nous reste à examiner leur place au sein de notre questionnement sur les fondements de la relation éducative, plus précisément sur le postulat d'éducabilité. Rappelons que la présente démonstration s'établit sous la forme d'un syllogisme.

\section{A.1 Qu'est-ce que le postulat d'éducabilité ?}

17 Le postulat d'éducabilité, autrement dit le postulat chez l'éducateur que toute personne dont il a la responsabilité possède toujours une aptitude à être éduquée et à progresser, se situe au fondement de l'acte éducatif. Si le terme d'éducabilité est relativement récent ${ }^{11}$, l'idée que l'être humain est perfectible et éducable est bien présente depuis les réflexions des philosophes de l'Antiquité grecque. Elle sera reprise et développée par plusieurs penseurs, parmi lesquels Comenius, Kant ou encore Rousseau qui insiste sur la «faculté de se perfectionner" de l'être humain, faculté "sur laquelle il ne peut y avoir de contestation» (1755/1981, p. 54). Toutefois, concernant Rousseau, de sérieuses réserves peuvent être émises quant à l'ériger en tant que héraut moderne du postulat d'éducabilité, comme nous y invite la lecture des lignes suivantes, issues du chapitre premier de l'ouvrage Émile ou De l'éducation:

Celui qui se charge d'un élève infirme et valétudinaire change sa fonction de gouverneur en celle de garde-malade ; il perd à soigner une vie inutile le temps qu'il destinait à en augmenter le prix [...] Je ne me chargerais pas d'un enfant maladif et cacochyme, dût-il vivre quatre-vingts ans. Je ne veux point d'un élève toujours inutile à lui-même et aux autres, qui s'occupe uniquement à se conserver, et dont le corps nuise à l'éducation de l'âme. Que ferais-je en lui prodiguant vainement mes soins, sinon doubler la perte de la société et lui ôter deux hommes pour un? (Rousseau, 1762/1966, p. 58).

Imaginons en effet une situation au sein de laquelle l'éducateur serait intimement persuadé que toute tentative de sa part pour mener le sujet éduqué vers un état qu'il estime préférable serait vouée à l'échec, perdue d'avance, l'élève étant, pour reprendre les mots de Rousseau, "toujours inutile à lui-même et aux autres». Dans une telle situation, l'avènement d'une relation éducative est impossible, tel que le fait nettement apparaitre le philosophe Alain dans le passage suivant :

Si je crois que l'enfant que j'instruis est incapable d'apprendre, cette croyance écrite dans mes regards et dans mes discours le rendra stupide; au contraire, ma confiance et mon attente est comme un soleil qui mûrira les fleurs et les fruits du petit bonhomme [...]. Il faut essayer, il faut croire (Alain, 1952, p. 227).

En s'appuyant sur certaines analyses de Philippe Meirieu (1991/2016, p. 34), Alain Trouvé (2011) souligne que l'éducabilité d'autrui, en ce qu'elle n'est pas véritablement quantifiable ni vérifiable de manière empirique à proprement parler, relève d'abord d'un postulat. L'auteur rappelle que le verbe postuler, du latin postulare, signifie «demander qu'on accorde ». En ce sens, postuler c'est demander et souhaiter que l'on puisse admettre de façon hypothétique, autrement dit en créditant la proposition de façon aprioriste. Aussi, l'éducabilité est d'abord postulée; en tant que postulat, "sa finalité ne consiste 
donc pas à rendre compte de la réalité, mais de la transformer autant qu'il est possible dans le sens d'une autonomisation de celui que l'on éduque» (Trouvé, 2011, p. 121). Autrement dit, quoi de plus pratique et concret qu'un postulat moral énoncé a priori ?

Pour l'éducateur, postuler l'éducabilité réside donc dans le «faire comme si 》 (Meirieu, 1991/2016, p. 45) la progression était toujours possible ; faire comme si les difficultés et obstacles auxquels se confronte le sujet éduqué étaient dépassables et temporaires. C'est donc avoir «l'exigence du meilleur », et «l'acceptation du pire» (p. 81). Postuler que la personne à éduquer est éducable amène ainsi à reconnaître en elle un potentiel qui ne saurait se réduire à la fatalité des déterminismes, à la considérer comme relationnelle, en construction et perfectible. Plus encore, le postulat d'éducabilité pourrait bien relever, d'une certaine manière, du domaine de la croyance et de la foi en l'autre, comme l'a mis en évidence Alain Trouvé :

[...] l'éducabilité ne peut qu'être objet de croyance. Cela veut dire qu'il faut la considérer comme un postulat moral, un principe pratique inconditionnel et indiscutable. [...] Si je crois le projet possible, je chercherai dans le réel des points d'appui pour mon action. Et puisque le réel est un immense réservoir de faits, je finirai toujours par en trouver (Trouvé, 2011, p. 122).

21 Aussi éduquer revient-il à développer confiance et foi en l'autre, en tout autre, à croire en ses possibilités de développement; plus généralement, à avoir confiance dans le processus éducatif. La foi dont il s'agit n'étant pas nécessairement religieuse, mais pouvant tout à fait être laïque. Le postulat d'éducabilité est donc un présupposé valide d'ordre logique, mais aussi éthique : il engage la responsabilité de l'éducateur, met en jeu son désir d'éduquer et une forme de foi en l'humanité. Il apparaît comme étant un pari sans doute non suffisant, mais tout à fait nécessaire pour entrer en relation éducative et cheminer progressivement vers l'émergence relationnelle du sujet éduqué comme personne.

\section{A.2 Difficultés, limites et pièges potentiels liés au postulat d'éducabilité}

Cependant, comme tout pari, le postulat d'éducabilité implique aussi l'acceptation de certains risques; risque d'échec du sujet éduqué, en particulier, ou risque d'échec de la relation si par exemple celui-ci résiste à ce que l'éducateur veut lui transmettre. De façon symétrique, une attente démesurée de résultat vis-à-vis de la réussite des sujets éduqués peut également entraîner, chez l'éducateur, une dérive vers le sentiment de toutepuissance. Cette dérive est bien pointée par Philippe Meirieu (1991/2016, 1993) comme " tentation démiurgique ", qui vise à faire de l'autre un objet que l'on façonne à sa guise. Tentation d'une attente démesurée envers l'autre et envers soi-même: "Nous ne supportons plus, alors, que l'autre nous échappe et nous nous tendons tout entier dans un désir effréné de maîtrise ", où en extrême limite pourrait naître l'« obsession de séduire ou de briser ", de punir ou d'humilier «celui qui se dérobe à notre pouvoir, avant de retourner la violence contre nous, en une culpabilité mortifère où l'échec de l'autre devient notre échec au point de compromettre jusqu'à notre raison d'être et d'enseigner » (Meirieu, 1991/2016, p. 42). Deux dangers menacent alors l'acte d'éduquer : celui de considérer l'autre comme un objet à fabriquer selon ses désirs (ce qui tire l'éducation du côté du dressage ou de la manipulation), ou celui, inverse et symétrique, de devenir indifférent à son égard, par dépit ou résignation, ou plus obscurément "par crainte d'être entraîné vers la destruction de l'autre et de [soi]-même ». On peut ainsi en 
arriver, paradoxalement, à détruire en soi « tout intérêt pour l'autre » (p. 42), à récuser l'éducabilité elle-même ; et donc toute possibilité d'instauration d'une relation éducative.

Modeler ou dresser un sujet, c'est restreindre sa liberté, tandis que l'attente du maître l'astreint à la reconnaissance obligatoire, et à la culpabilité si cette reconnaissance n'est pas suffisante; la tentation du dressage revient alors tout simplement à «nier l'éducation ", à "prendre le parti du conditionnement " et de la captation totalitaire, autrement dit, donc, à confondre l'éducation d'un sujet émancipé avec la fabrication d'un objet à s'approprier ou à marchander. Cela nous rappelle à quel point la tentation de réification d'autrui est présente dans une relation asymétrique de pouvoir, et à quel point il est primordial de considérer celui que l'on éduque avant tout comme un sujet, et audelà, de considérer tout être humain sans exception en tant que Personne - fin en soi, injonction au respect inconditionnel de la dignité, ce qui interdit toutes formes de nuisance et de violence à son égard; car la personne n'est pas un objet que l'on construit, mais bien un sujet qui se co-construit de manière relationnelle, et qui demeure toujours singulier et inachevé dans ses dimensions plurielles (par exemple morale, cognitive, intellectuelle, sociale, citoyenne, physique, langagière, artistique, psychologique voire

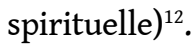

Accepter de renoncer à cette forme de captation repose donc sur « la capacité à intégrer la négativité de l'éducabilité » (Meirieu, 1991/2016, p. 45), c'est-à-dire le risque de l'échec de la relation, de l'éduqué, de l'éducateur. Postuler l'éducabilité implique de reconnaître et d'accepter ce risque, et c'est, toujours d'après Philippe Meirieu, «sans aucun doute, la chose la plus difficile du monde» (p. 45). D'autant que cette négativité potentielle ne se limite pas, selon nous, à ce risque, car il peut arriver parfois que l'éduqué réussisse si bien, ou mette tant ses efforts au service de la réussite, qu'il en devienne en quelque sorte gênant pour l'éducateur, car trop proche, trop identique à lui - car trop installé dans son désir. Quand l'élève dépasse le maître, ou en prend le chemin, c'est un autre versant de la négativité qui peut se dévoiler. Or lorsque l'éducateur tombe dans le piège du désir de maîtrise, de captation, il s'agit au fond, vraisemblablement, de symptômes d'une maladie d'attachement à son moi (ego). Si je désire absolument que l'autre suive mes pas et le projet que j'ai dessiné pour lui, je désire qu'il me ressemble, qu'il m'imite dans mes efforts et dans mes réussites, qu'il incarne l'idéal que j'ai forgé pour moi et pour lui. De fait, imiter l'autre est constitutif de la relation humaine ; s'éduquer et apprendre, cela consiste souvent à imiter le maître, dans ses actes et dans ses préférences, dans les objets qu'il désigne, dans son désir en somme.

Ce phénomène et ses risques sont étudiés dans le cadre de l'anthropologie mimétique de René Girard, et de ce qu'il nomme « le désir selon l'autre », désir « métaphysique » dans la mesure où selon lui «tout désir est désir d'être » (1994, p. 28). L'éducateur se pose ainsi comme un médiateur du désir de l'éduqué vers des modes d'être aussi bien que vers des objets de savoir désignés comme désirables, par le seul fait qu'il se présente comme les désirant lui-même. Le piège qui guette ce médiateur est notamment de tomber dans la complaisance d'une fascination qu'il exerce :

Le maître est ravi de voir les disciples se multiplier autour de lui, il est ravi de se voir pris comme modèle. Néanmoins, si l'imitation est trop parfaite, si l'imitateur menace de surpasser le modèle, voilà le maître qui change systématiquement d'attitude et commence à se montrer méfiant, jaloux, hostile. Il va faire tout ce qu'il pourra pour déprécier le disciple et le décourager (Girard, 1978, pp. 410-411). 
imésis qui est légitime, celle qui permet d'apprendre et de s'élever, peut parfois se pervertir en une mimésis violente, parce que rivalitaire. À trop encourager l'imitation, l'éducateur fait ainsi courir le risque de faire basculer la mimésis d'apprentissage vers la mimésis de rivalité (p. 410). Et renoncer à cette forme d'antagonisme n'est pas facile, car lorsqu'on veut être tout pour l'autre, on a en même temps besoin de cet autre pour exister, même dans l'affrontement, et même dans l'exercice d'une domination - ne seraitce que pour rester en présence de sa propre raison d'éduquer, voire d'exister. Du côté du sujet éduqué, une telle relation court alors le risque de se vivre comme une recherche de mise en conformité de soi-même avec le désir de l'éducateur, ce qui expose au «double bind inextricable de l'imitation qui se retourne contre l'imitateur alors que le modèle et la culture entière l'invitent expressément à imiter » (p. 411).

Ce risque nous invite à considérer avec attention la question de la réciprocité dans la relation éducative; de quelle "réciprocité » parle-t-on entre l'éducateur et le sujet éduqué ? Doit-elle exister et à quelles conditions? Comment éviter que la «bonne» réciprocité ne glisse vers la «mauvaise »? Faut-il prévenir les uns et les autres contre le «double impératif qui vient de l'autre en tant que modèle - imite-moi - et en tant que rival - ne m'imite pas » (p. 411) ? À sa manière, Emmanuel Lévinas souligne lui aussi les dangers potentiels de la réciprocité dyadique dans un entretien portant sur la question éducative :

La réciprocité introduit un élément qui me semble dangereux. Chez Martin Buber, pour ne citer que lui, le $\mathrm{Je} / \mathrm{Tu}$ est une relation dans laquelle chacun est la condition de reconnaissance de l'autre. Je suis par rapport à toi, tu es par rapport à moi. Rien n'est plus opposé à ma conception des relations interhumaines. Pour moi, l'essentiel est dans le débordement de cette relation. On ne présente pas tout de suite la facture. [...] Je ne dois rien attendre d'autrui (Lévinas, 1996, pp. 120-121).

Autant de mises en garde contre les dangers éventuels de la réciprocité dans la dyade $\mathrm{Je} / \mathrm{Tu}$ qui nous enjoignent à nous interroger sur les façons de les éviter, à trouver des antidotes permettant de ne pas tomber " malade de l'éducabilité » (Meirieu, 1991/2016, p. 41). Pour ce faire, Philippe Meirieu théorise le principe de non-réciprocité, qu'il associe au principe de liberté.

\section{A.3 Quels antidotes pour déjouer les pièges potentiels ? Du principe de non-réciprocité à l'altruisme}

Il semble légitime de penser que le dévoiement potentiel du postulat d'éducabilité, une fois dans la pratique éducative concrète, relève autant d'une dérive que d'une forme de méprise fondamentale s'il est envisagé, dès le départ, de manière confuse.

Nous avons vu que la tentation de toute-puissance peut guetter l'éducateur, et ce d'autant plus que la relation éducative est asymétrique, asymétrie ne concernant bien sûr que les savoirs et les compétences des acteurs, et non pas les Personnes dans leur dignité. Le principe de non-réciprocité suppose que l'éducateur donne aux personnes qu'il éduque sans rien attendre d'elles en retour. Il consiste à parier sur l'émancipation d'autrui, et à agir selon cette finalité, sans pour autant être dans l'attente d'une quelconque reconnaissance personnelle de la part d'autrui : en d'autres termes, on n'éduque pas pour être remercié en contrepartie de sa foi, de sa confiance et de ses efforts. On n'éduque pas non plus pour parvenir à un soi-disant "résultat » fixé à l'avance, ce qui rejoindrait le façonnage d'objet évoqué plus haut. Au contraire, la personne relationnelle, singulière et 
toujours inachevée, qui émerge progressivement dans la relation éducative, devra toujours échapper aux diverses formes de manipulation ou de dressage. Il s'agit de lui permettre de cheminer vers davantage de liberté intérieure, d'autonomie, vers un mieuxêtre. Éduquer, écrit Anne-Marie Drouin-Hans, c'est donc "faire le deuil de la réussite " (2009, p. 42), dans la mesure où « une réussite programmée est contradictoire avec l'idéal d'une éducation libératrice » (p. 43).

Le pari de l'éducabilité nous reconduit donc vers la nécessité de distinguer la praxis (qui trouve sa fin en elle-même et ne cesse pas quand cette fin est atteinte) de la poïesis (la fin est extérieure à l'activité, et cesse quand l'objectif est atteint) ; « continuer à parier sur la réussite d'autrui, tout faire pour y parvenir, mais sans exiger d'être payé en retour dans un rapport de réciprocité marchande : telle est bien la condition pour qu'un obscur projet de maîtrise accède à la position éthique » (Meirieu, 1991/2016, p. 45). Il s'agit d'accepter en fin de compte que ce postulat puisse être mis en échec, sans pour autant y renoncer. En ce sens, l'éthique de la relation éducative est bel et bien contraire à l'idéologie néolibérale de type marchand qui investit sur un produit et attend une plus-value en retour. Éduquer consiste à souhaiter l'épanouissement du sujet éduqué, son développement, son émancipation, jusqu'à ce qu'il puisse voler de ses propres ailes, voire peut-être dépasser le maitre, et à s'en réjouir le moment venu (Terraz, 2017b). C'est savoir se libérer de l'attente, agir dans une certaine équanimité, libre de l'attachement qui rapproche des uns et de la répulsion qui éloigne des autres. Car avoir trop d'attentes, c'est s'exposer à la déception qui mène au dépit :

[...] c'est, sans doute, quand l'éducateur a trop attendu ou, plus exactement qu'il a attendu la réponse de l'autre comme son dû, que, par dépit, il affecte de ne plus rien attendre. C'est, sans doute, quand on n'a pas intégré le principe de non-réciprocité que l'on adopte le principe d'indifférence, en faisant mine de croire que c'est exactement la même chose (Meirieu 1991/2016, p. 50).

Le principe de non-réciprocité s'articule également avec le principe de liberté, qui souligne l'importance d'un engagement mutuel et libre de la part de chacun des acteurs de la relation éducative. Éduquer, c'est reconnaitre et respecter le sujet éduqué comme Personne dans ses droits, dans sa liberté, tout en visant son émergence comme personne aussi libre et heureuse que possible. Le postulat d'éducabilité est donc nécessaire - mais non suffisant - pour accéder à une relation véritablement éducative, entendue dans le sens d'une influence libératrice et non d'une influence manipulatrice; pour le dire autrement, c'est une influence qui travaille à sa propre cessation. On perçoit aisément en quoi le principe de non-réciprocité renvoie à une éthique de l'altruisme : donner sans rien attendre en retour, respecter autrui dans sa liberté et accepter l'autre avec ses qualités et ses faiblesses. Comme énoncé précédemment, l'altruisme se distingue radicalement de l'amour éros, et ne saurait en aucun cas se confondre avec lui : il ne consiste pas à aimer pour ce que l'autre peut nous apporter, dans un rapport de séduction ou de fusion relationnelle, mais au contraire à souhaiter son bonheur et sa liberté et à agir selon cette intention. L'altruisme se distingue donc de l'attachement affectif ou émotionnel, il ne vise pas la réciproque mais pourrait permettre d'instaurer une juste et bonne réciprocité relationnelle, ouverte au tiers, capable de déjouer les pièges de la réciprocité dyadique. 


\section{[Prémisse B] L'altruisme permet au postulat d'éducabilité de se mettre en mouvement de façon juste et bonne, de passer du désir à l'action d'éduquer}

À ce stade de notre analyse, reste à savoir comment passer du postulat d'éducabilité à l'agir éducatif, du principe à l'acte. Car les principes, valeurs et devoirs les plus louables sont condamnés à demeurer inactifs si l'agent moral n'a pas le désir d'y tendre et de les appliquer dans les situations singulières; ou encore s'il ne sait pas comment s'y prendre de façon concrète ${ }^{13}$. En quoi et pourquoi l'altruisme de l'éducateur est-il nécessaire pour que le postulat d'éducabilité puisse prendre corps de façon juste et bonne dans la relation éducative et, ainsi, jouer un rôle de garde-fou et d'antidote face aux écueils et pièges potentiels?

\section{D'où vient le désir d'éduquer et d'agir dans la relation ? Lumières de l'allégorie de la caverne}

Sous une forme métaphorique d'une puissance heuristique remarquable, construite autour d'un dialogue entre Socrate et Glaucon, cette allégorie (Platon, La République, livre VII) traite de l'éducation des philosophes destinés, dans la perspective platonicienne, à gouverner la cité. Les esclaves prisonniers de la caverne, qui «nous ressemblent " (515a) précise le texte, se trouvent initialement dans un état d'illusion et d'ignorance de leur propre état de souffrance. En décrivant le cheminement d'un esclave depuis la caverne jusqu'au monde des Idées, cheminement qui symbolise le passage de l'illusion vers la connaissance de la vérité des choses telles qu'elles sont, Platon montre en quoi la sortie progressive de la caverne est une aventure humaine difficile et semée d'embûches, une "montée rude et escarpée " (516a) mais possible. Lorsque l'ancien prisonnier prend conscience de l'état d'illusion dans lequel il se trouvait, il s'en " félicite", se réjouit de sa belle et nouvelle condition, et prend alors les autres «en pitié » (516c). Une fois sorti lui-même de l'ignorance fondamentale dans laquelle il se trouvait, il devient éducateur et décide librement, en conscience et raison, de prendre la responsabilité de retourner dans la caverne pour en faire sortir les prisonniers restés à l'intérieur. En d'autres termes, il prend le risque de retourner dans l'obscurité, de s'exposer aux railleries de ses compagnons, et fait le choix d'agir pour les aider à sortir de l'ignorance et de la souffrance : ne pourrait-on pas qualifier cette intention d'altruiste?

Cette allégorie permet de mettre en évidence que ce qui met l'éducateur en tant qu'agent moral en mouvement vers autrui, c'est d'abord la prise de conscience de l'ignorance et de la souffrance d'autrui comme de soi-même. Platon montre qu'il est nécessaire de sortir soi-même de la caverne pour voir naître en soi le désir d'en libérer les autres, en les aidant à redécouvrir ce qui était déjà présent en germe chez chacun d'entre eux. Si l'on est trop ignorant de son propre état d'illusion et de souffrance, on ne peut se tourner véritablement vers autrui pour l'aider à s'émanciper. Cette allégorie met donc bien en évidence le sens qu'il y a à postuler que chacune de ces personnes est éducable.

De façon plus générale, il nous semble que c'est en situant la réflexion au sein d'une « ontologie relationnelle de l'interdépendance » (Terraz, 2018a, à partir de Jacques, 1982) que l'on serait le mieux à même de saisir les enjeux liés à la relation éducative, 
interpersonnelle et interlocutive, donc aussi d'éclaircir la question de l'articulation entre le postulat d'éducabilité et l'altruisme. Lorsque l'éducateur prend véritablement conscience de la nature interdépendante de l'être humain, de la sienne comme de celle des personnes qu'il éduque, il devient plus à même de se décentrer du « moi » (ou ego) pour s'ouvrir de façon libre et confiante dans la relation avec, par et pour autrui. Postuler l'éducabilité d'autrui, de tout autrui, c'est reconnaitre sa dimension relationnelle et interdépendante, c'est toujours le considérer en tant que Personne (comme fin en soi) et en tant que personne relationnelle (comme finalité éducative).

Ce caractère relationnel et interdépendant relève de toute façon de la condition humaine, car dès sa naissance l'être humain apparaît comme incomplet, autrement dit néotène : il ne peut se développer ni s'émanciper sans être en relation avec d'autres qui l'initient au monde, lui prodiguent des soins, le protègent, et ne peut se développer non plus sans une éducation qui autorise la co-construction progressive de savoirs, de savoir-faire et de savoir-être. Cet inachèvement fondamental fait de lui un être perfectible et donc potentiellement éducable. L'éducation est donc, ainsi que Kant (1776-1787/2004) n'eut de cesse de le rappeler, une nécessité anthropologique. Ce qui fait du postulat d'éducabilité un postulat valide du point de vue anthropologique, éthique, mais aussi ontologique. Bien entendu, le postulat d'éducabilité ne consiste pas à nier les possibles différences qui existent entre les personnes quant à leur potentiel et leurs capacités d'apprentissage, ni à dénier tout obstacle à venir dans la relation éducative. De plus, si la perfectibilité de l'être humain est bel et bien un constat anthropologique, Eirick Prairat souligne que le postulat d'éducabilité ne s'y limite pas : il consiste plutôt à énoncer que « Paul est éducable ici et maintenant et ce grâce à moi, et partant, il en est de même pour Pauline, Pierre, Jacques et tous les autres » (Prairat, 2014, p. 123).

S'inscrire dans une ontologie relationnelle de l'interdépendance revient aussi à prendre conscience de la non-totalité de l'être, de son caractère dynamique et ouvert à la relation. Cette prise de conscience n'étant pas évidente, cela pourrait expliquer que nous nous trouvions le plus souvent dans un état d'insatisfaction chronique, ce qui pourrait rejoindre la conception de l'être humain chez Paul Ricœur comme être «agissant et souffrant » (1990, p. 29, p. 172). On retrouve d'ailleurs ici la dimension éthique inhérente à l'action éducative, qui apparait avec la double étymologie latine du mot « éducation » : le verbe educare signifie se soucier de l'autre, en prendre soin en l'aidant à grandir, à se développer, à s'instruire, et le verbe ex-ducere signifie conduire hors de, guider hors de ; l'idée est bien d'aider, autant que faire se peut, le sujet éduqué à s'éloigner de l'ignorance et de la souffrance. Au fond, le désir d'éduquer entre peut-être véritablement en jeu lorsque l'éducateur est en mesure de reconnaître l'état d'ignorance et de souffrance dans lequel se trouvent le sujet éduqué mais aussi lui-même, et qu'il souhaite agir pour aider l'autre à s'en éloigner. Insistons à nouveau sur le fait que reconnaître ses propres carences, faiblesses, difficultés, et développer de la sollicitude à l'égard de soi-même est fondamental pour être en capacité de prendre soin d'autrui.

\section{[Conclusion] L'altruisme est nécessaire mais non suffisant pour construire une relation éducative}

En tirant les fils de notre argumentation logique, nous parvenons à la conclusion selon laquelle l'altruisme est nécessaire mais non suffisant pour construire une relation éducative. Il ne peut y avoir d'éducation sans altruisme, sauf à forcer le sens des mots. 
Qu'une telle conclusion puisse relever du simple bon sens est peut-être davantage une force qu'une faiblesse; car réinterroger voire réaffirmer ce qui semble relever de l'évidence, en prenant soin d'éclairer les confusions potentielles, est l'une des fonctions de la philosophie.

Nous avons montré que la notion de bienveillance, en tant que qualité humaine, apparaît féconde pour cerner ce qui va porter l'agent moral dans son choix d'intérioriser des postulats et principes comme le postulat d'éducabilité. Pour autant, nous avons aussi montré en quoi elle n'est vraisemblablement pas suffisante pour désigner le processus de mise en mouvement de l'agent moral, qui décide librement, en conscience et raison, d'incarner et d'appliquer le postulat d'éducabilité dans l'action relationnelle éducative. Aussi avons-nous introduit le concept d'altruisme en vue de compléter et de renforcer la notion de bienveillance, en tant que l'altruisme est inconditionnel, désintéressé, traduit en acte, et qu'il permet d'instaurer une juste et bonne distance entre chacun des acteurs dans la singularité de chaque relation éducative.

41 En associant l'altruisme et le postulat d'éducabilité, nous donnons une dimension inconditionnelle à ce dernier : non seulement je postule que le sujet éduqué qui me fait face est éducable, mais j'intègre aussi dans ce postulat l'autrui universel. Tous les êtres humains sans exception sont interdépendants, néotènes, et souhaitent s'éloigner de l'ignorance et de la souffrance. Prendre conscience de cela et agir selon cette intention est un formidable rempart contre la violence, notamment contre l'exclusion victimaire du tiers personnel. Lorsqu'il est rendu possible et mis en application à travers une éthique éducative de l'altruisme, le postulat d'éducabilité prend donc une dimension à la fois singulière et universelle qui permet de ne laisser aucun des sujets éduqués sur le bord du chemin. L'autrui universel, tel que défini, n'est pas une abstraction ni de l'ordre de l'impersonnel: il caractérise soi-même et l'autre singulier, la ou les personnes qui sont présentes concrètement dans une relation de proximité, mais il ne s'y limite pas. L'éducation se dépasse elle-même; on éduque aussi pour les générations futures, pour l'autre comme tiers, pour celui, celle et ceux qui ne sont pas là, ou pas encore là.

42 La personne qui s'engage dans une relation véritablement éducative est toujours animée par la bienveillance et l'altruisme ; elle dispose d'un potentiel qu'elle seule peut décider d'actualiser, de faire croître toujours davantage. Cet engagement relève d'un choix libre et personnel de la part de l'éducateur, qui s'établit, comme nous l'avons dit, à partir d'une double prise de conscience : celle de l'état initial d'ignorance et de souffrance dans lequel se trouvent le sujet éduqué comme lui-même, et celle de la dimension relationnelle et interdépendante de l'être humain. Le processus éducatif est une relation humaine vivante de personne à personne, et l'éducateur un agent moral, un praticien réflexif de l'éthique. L'éthique éducative impose alors de trouver un équilibre entre le piège du nihilisme et celui du moralisme dogmatique. En ce qu'elle s'appuie sur le respect inconditionnel de la Personne dans sa liberté et sa dignité, une éthique éducative de l'altruisme ne relève pas d'un moralisme paternaliste, et s'il existe inévitablement une certaine forme de paternalisme dans la relation éducative, il devrait s'agir, comme le souligne Eirick Prairat, d'un « paternalisme faible » (2013, pp. 126-127).

43 Faire le choix d'éduquer revient finalement à avoir foi en l'être humain, et à faire naître un désir d'agir en vue de permettre à l'humanité, dans ce qu'elle a de plus singulier et de plus universel, d'être un peu plus libre, un peu plus émancipée, un peu plus éclairée, et, ne craignons pas de le dire, un peu plus heureuse. Il ne s'agit pas, sur ce dernier point, d'imposer au sujet éduqué sa propre conception du bonheur, mais de réunir les 
conditions favorisant son émergence comme personne capable de trouver son propre chemin vers un mieux-être. Autant de finalités éducatives déjà énoncées par Emmanuel Kant, auquel nous donnerons la parole pour conclure et ouvrir notre réflexion :

« [c]'est une chose enthousiasmante de penser que la nature humaine sera toujours mieux développée par l'éducation et que l'on peut parvenir à donner à cette dernière une forme qui convienne à l'humanité. Ceci nous ouvre une perspective sur une future espèce humaine plus heureuse » (Kant, E., 1776-1787/2004, pp. 100-101).

\section{BIBLIOGRAPHIE}

Alain (1952). Propos d'un Normand I. Paris : Gallimard.

Aspy, D. \& Roebuck, F. (1990). On n'apprend pas d'un prof qu'on n'aime pas. Résultats de recherches sur l'éducation humaniste (Trad. L.-B. Lalanne). Montréal : Actualisation. Ouvrage original paru sous le titre Kids don't learn from people they don't like.

Centre national de ressources textuelles et lexicales (CNRTL), entrée « bienveillance ». Repéré à http://www.cnrtl.fr/definition/bienveillance.

Collectif (1994). Le Robert pour tous. Paris : Le Robert.

Drouin-Hans, A.-M. (1998). L'Éducation une question philosophique. Paris : Anthropos/Economica, coll. «Éducation Poche ».

Drouin-Hans, A.-M. (2004). Valeurs et culture(s) : peut-on encore penser l'universel ? Tréma, 23 («Approche anthropologique en éducation et en formation I »). Repéré à http:// trema.revues.org/640 ; DOI : http://dx.doi.org/doi:10.4000/trema.640.

Drouin-Hans, A.-M. (2009). L'éducation au cœur de l'autorité. Le Télémaque, 35, 41-48. Caen : Presses universitaires de Caen. Repéré à http://www.cairn.info/revue-le-telemaque-2009-1page-41.htm ; DOI : http://dx.doi.org/doi:10.3917/tele.035.0041.

Girard, R. (1978). Des choses cachées depuis la fondation du monde. Paris : Grasset.

Girard, R. (1994). Quand ces choses commenceront. Paris : Arléa.

Godin, C. (2004). Dictionnaire de philosophie. Paris : Fayard.

Jacques, F. (1982). Différence et subjectivité. Anthropologie d'un point de vue relationnel. Paris : Aubier Montaigne, coll. « Analyse et raisons ».

Jacques, F. (2007). L'arbre du texte et ses possibles. Paris : Librairie philosophique J. Vrin, coll. «Problèmes \& Controverses ».

Jennings, P. A. \& Greenberg, M. T. (2009). The Prosocial Classroom: Teacher Social and Emotional Competence in Relation to Student and Classroom Outcomes. Review of Educational Research, 79 (1), 491-525. Repéré à http://journals.sagepub.com/doi/abs/10.3102/0034654308325693 ; DOI :

http://dx.doi.org/doi:10.3102/0034654308325693.

Kant, E. (1994). Métaphysique des mœurs II. Doctrine du droit. Doctrine de la vertu. (Trad. A. Renaut ; 1797). Paris : GF Flammarion. 
Kant, E. (2004). Réflexions sur l'éducation (Trad. A. Philonenko ; $8^{\mathrm{e}}$ édition, ouvrage original publié à partir de cours dispensés entre 1776 et 1787). Paris : Librairie philosophique J. Vrin.

Lecomte, J. (2012). La bonté humaine, altruisme, empathie, générosité. Paris : Odile Jacob.

Lévinas, E. (1996). Entretien. Dans A. Hocquard (dir.), Éduquer, à quoi bon ? Ce qu'en disent philosophes, anthropologues et pédagogues (pp. 119-123). Paris : PUF, coll. « L'éducateur ».

Martinez, M.-L. (1997). Vers la réduction de la violence à l'école. Contribution à l'étude de quelques concepts pour une anthropologie relationnelle de la personne en philosophie de l'éducation. Thèse de doctorat sous la direction de Francis Jacques. Université de Paris III - Sorbonne Nouvelle. Lille : Septentrion. Version en ligne (1996) repérée à https://tel.archivesouvertes.fr/tel-00201180/.

Martinez, M.-L. (1999). L'intégration du tiers personnel, une valeur au-dessus de tout soupçon pour l'éducation. Dans S. Solère-Queval (dir.), Les valeurs au risque de l'école (pp. 159-169). Villeneuve-d'Ascq : Presses universitaires du Septentrion.

Martinez, M.-L. (dir.) (2002). L'émergence de la personne. Éduquer, accompagner. Paris : L'Harmattan, coll. «Crise et Anthropologie de la relation ».

Meirieu, P. (1993). L'envers du tableau. Quelle pédagogie pour quelle école ? Paris : ESF Éditeurs.

Meirieu, P. (2016). Le choix d'éduquer, éthique et pédagogie (12éd. ; 1991). Paris : ESF Éditeurs.

Platon. La République (Trad. E. Chambry). Paris : Gallimard, coll. « Tel » (Société d'édition « Les Belles Lettres ", 1989 pour les livres I à VII).

Prairat, E. (2013). La morale du professeur. Paris : PUF.

Prairat, E. (2014). Questions de discipline à l'école et ailleurs... Toulouse : Érès.

Prairat, E. (2017). Éduquer avec tact. Vertu et compétence de l'enseignant. Paris : ESF Sciences Humaines, coll. «Pédagogies Questions Vives».

Ricœur, P. (1990). Soi-même comme un autre. Paris : Éditions du Seuil, coll. « Points Essais ».

Rousseau, J.-J. (1966). Émile ou De l'éducation (1762). Paris : GF-Flammarion.

Rousseau, J.-J. (1981). Discours sur l'origine et les fondements de l'inégalité parmi les hommes (1755). Paris : Nathan.

Terraz, T. (2016). Le care en éducation et la posture éthique de l'enseignant : une place pour la valeur-actitude altruisme ? Éducation et socialisation, Les cahiers du CERFEE, 40 (« Le care en éducation : quelle(s) reconfiguration(s) ? »), PULM. Repéré à http://edso.revues.org/1526 ; DOI : http://dx.doi.org/doi:10.4000/edso.1526.

Terraz, T. (2017a). Discours politiques de la bienveillance et éthique de l'altruisme en éducation. Dans M. Fabre, H.-L. Go et E. Prairat (dir.), Éthique et politiques éducatives (pp. 151-166). Nancy : Presses universitaires de Nancy - Éditions universitaires de Lorraine, coll. «Questions d'éducation et de formation ».

Terraz, T. (2017b). L'altruisme, une condition de l'autorité éducative émancipatrice ? Recherches en éducation, 30, novembre ("Recherches en éducation et formation : contributions des doctorants et jeunes chercheurs ", numéro coordonné par S. Besnier, S. Joffredo Le Brun, V. Messina, C. Riban et M. Roche), 39-49. Repéré à http://www.recherches-en-education.net/ spip.php?article362.

Terraz, T. (2017c). Le savoir en éducation : entrevoir la relation d'interdépendance au-delà des confusions. Questions vives, Recherches en éducation, 28 (" De l'indifférenciation à la différenciation des domaines du savoir et du symbolique », dossier coordonné par M.-L Martinez \& E. Annoot). 
Éditeur : Université Aix-Marseille. Repéré à http://journals.openedition.org/questionsvives/2803 ; DOI : http://dx.doi.org/doi:10.4000/questionsvives.2803.

Terraz, T. (2018a). L'engagement éthique par et pour la personne : altruisme et décentrement du moi. Dans M. Janner-Raimondi \& A. Trouvé (dir.), L'engagement éthique en éducation et en recherche (pp. 53-77). Rouen : PURH, coll. « Penser les valeurs en éducation et en formation ».

Terraz, T. (2018b). Penser l'articulation entre vertu et devoir avec Eirick Prairat : analyse et discussion méta-éthique. Dans H.-L. Go (dir.), Éthique et éducation : Questions à Eirick Prairat (pp. 175-195). Rouen : PURH, coll. «Penser les valeurs en éducation et en formation ».

Terraz, T. (2018c, à paraître). L'altruisme au cœur des conditions de l'Éducation. Éthique de la relation éducative et émergence de la personne : investigation philosophique. Thèse de doctorat soutenue le 30 novembre 2018 à l'université « Rouen Normandie » et réalisée sous la direction de Madame Marie-Louise Martinez et de Monsieur Eirick Prairat.

Trouvé, A. (2011). « Croire » : Alain, Meirieu ou la foi (sans foi) du pédagogue. Dans M. Soëtard et G. Le Bouëdec (dir.), La foi du pédagogue. Fondements - Figures - Pratiques (pp. 109-125). Paris :

Éditions Don Bosco, coll. «Sciences de l'éducation ».

\section{NOTES}

1. Par exemple Terraz $(2016,2017 \mathrm{a} / \mathrm{b}, 2018 \mathrm{a} / \mathrm{b} / \mathrm{c})$

2. Nous empruntons cette expression d'« émergence de la personne » aux travaux de Martinez (2002).

3. Dans l'ouvrage de Philippe Meirieu (1991/2016) portant sur l'éthique de l'éducateur et sur le postulat d'éducabilité, il nous semble percevoir en filigrane les dimensions de bienveillance et d'altruisme même si elles ne forment pas, à proprement parler, le cœur de son objet d'étude.

4. Nous faisons ici référence à l'ensemble des professionnels des métiers de l'éducation: enseignants, éducateurs spécialisés, conseillers principaux d'éducation, etc.

5. Francis Jacques précise par ailleurs que les textes scientifiques répondent au " problème », les textes littéraires et poétiques à l'« énigme », et les textes religieux et théologiques au "mystère » (2007, pp. 157-204).

6. CNRTL : http://www.cnrtl.fr/definition/bienveillance. Voir aussi dictionnaire Le Robert (1994, p. 110).

7. Nous reprenons ce concept à Francis Jacques (1982) et à Marie-Louise Martinez (1997, 1999).

8. Comme le dirait Eirick Prairat à propos de la vertu de tact (2017).

9. La notion d'«universalisable" avancée par Drouin-Hans $(1998,2004)$ et exposée précédemment nous semble être une voie favorable pour approcher l'idée d'héritage culturel et symbolique de l'humanité à la fois singulier et universel. En ce sens, postuler des universalisables, à réinterroger sans cesse, n'est pas une tentative de lissage des différences mais bien au contraire de dévoilement des richesses.

10. Voir par exemple l'ouvrage de Jacques Lecomte (2012), qui offre une importante revue de littérature sur cette question.

11. Le CNRTL (http://www.cnrtl.fr/definition/éducabilité) indique que le mot apparaît dès 1831 dans un ouvrage de Nodier.

12. Le lecteur souhaitant obtenir davantage de détails sur cette proposition réflexive de distinction entre les concepts de Personne (avec majuscule) et de personne pourra notamment se référer à Terraz $(2017 b, 2018 a)$. 
13. Aussi, d'un point de vue méta-éthique, il semble légitime de considérer que la vertu d'altruisme est une condition d'accès puis de juste et bonne mise en application des principes et devoirs moraux dans chaque situation particulière (Terraz, 2018b).

\section{RÉSUMÉS}

Cette étude, s'inscrivant dans le domaine de la philosophie de l'éducation, se propose d'interroger d'un point de vue réflexif, spéculatif et logique la bienveillance de l'éducateur au regard du postulat d'éducabilité. À la fois présupposé d'ordre logique et principe éthique, ce postulat demande de créditer de façon aprioriste et gratuite le sujet comme éducable. Il pourrait faire figure de point d'ancrage permettant la mise en œuvre d'une éthique de la bienveillance dans le champ de l'éducation et de la formation. Notre articulation entre la bienveillance éducative et le postulat d'éducabilité fera émerger le concept d'altruisme. La démonstration proposée, sous forme de syllogisme, amènera à considérer que l'altruisme, en ce qu'il est inconditionnel, intimement lié à l'action relationnelle et à l'intégration du tiers personnel/de l'autrui universel, est une condition nécessaire et non suffisante pour permettre l'avènement d'une relation véritablement éducative favorisant l'émergence du sujet éduqué comme personne dans ses dimensions plurielles.

This study, which is part of the field of the philosophy of education, aims to question from a reflexive, speculative and logical point of view the benevolence of the educator with regard to the postulate of educability. Both a logical assumption and an ethical principle, this postulate requires that the subject be credited a priori and gratuitously as an educable subject. It could serve as an anchor point for the implementation of an ethics of caring in the field of education and training. Our articulation between educational benevolence and the postulate of educability will bring out the concept of altruism. The proposed demonstration, in the form of a syllogism, will lead us to consider that altruism, in that it is unconditional, intimately linked to relational action and to the integration of the "tiers personnel »/ « autrui universel », is a necessary and not sufficient condition to allow the advent of a truly educational relationship favouring the emergence of the educated subject as a person in its plural dimensions.

\section{INDEX}

Mots-clés : altruisme, bienveillance, relation éducative, éthique éducative, postulat d'éducabilité

Keywords : altruism, benevolence, educational relationship, educational ethics, postulate of educability

\section{AUTEURS}

\section{TOMMY TERRAZ}

Docteur en Sciences de l'éducation, membre du laboratoire Cirnef de l'université « Rouen Normandie ", chercheur associé au laboratoire Lisec, membre de la Sofphied (Société 
francophone de philosophie de l'éducation). Son travail, en philosophie de l'éducation, consiste à interroger les conditions, les valeurs et les finalités de l'éducation, et porte sur la question d'une éthique éducative de l'altruisme.

\section{AMANDINE DENIMAL}

Maître de conférences en Sciences du langage au laboratoire Dipralang de l'université Paul Valéry Montpellier III. Ses travaux articulant didactique, épistémologie et anthropologie portent notamment sur la question du sujet dans la relation d'éducation/enseignement, et sur les axiologisations éducatives. 